\title{
Uncertainty quantification works relevant to fission yields and decay data
}

\author{
Go Chiba* and Shunsuke Nihira \\ Hokkaido University, Kita 13 Nishi 8, Kita-ku, Sapporo 060-8628, Japan
}

Received: 29 September 2017 / Received in final form: 21 January 2018 / Accepted: 14 May 2018

\begin{abstract}
In the present paper, firstly, we review our previous works on uncertainty quantification (UQ) of reactor physics parameters. This consists of (1) development of numerical tools based on the depletion perturbation theory (DPT), (2) linearity of reactor physics parameters to nuclear data, (3) UQ of decay heat and its reduction, and (4) correlation between decay heat and $\beta$-delayed neutrons emission. Secondly, we show results of extensive calculations about UQ on decay heat with several different numerical conditions by the DPTbased capability of a reactor physics code system CBZ.
\end{abstract}

\section{Introduction}

Generally there are two numerical procedures for uncertainty quantification (UQ) of reactor physics parameters induced by nuclear data uncertainty: the adjoint-based procedure and the stochastic-based procedure. In recent years, the stochastic-based procedure has been adopted frequently by virtue of drastic advancements of computer resources, but the adjoint-based procedure is still powerful because it can yield sensitivity profiles of reactor physics parameters with respect to nuclear data without any statistical fluctuations with short computation time relative to the stochastic-based procedure. Sensitivity profiles of reactor physics parameters to nuclear data are quite beneficial quantities to understand physical mechanism behind numerical results, and to identify important nuclear data for accurate estimations of reactor physics parameters.

Our research group in Hokkaido University has carried out several works about UQ based on the adjoint-based procedure. We have focused mainly on nuclide transmutation problems such as nuclear fuel depletion and $\beta$-delayed neutrons emissions, and have developed numerical tools based on the DPT. The present paper consists of two parts; the first part is to review our previous works on UQ based on $\mathrm{DPT}$, and the second part provides some numerical results of UQ for decay heat in various conditions using our tools.

\section{Reviews of UQ works done at Hokkaido University}

In the present section, our previous works on UQ are briefly reviewed.

\footnotetext{
* e-mail: go_chiba@eng.hokudai.ac.jp
}

\subsection{Development of numerical tools based on DPT}

The adjoint-based procedure requires sensitivity profiles of reactor physics parameters with respect to nuclear data, and these can be efficiently calculated by the perturbation or generalized perturbation theory. If reactor physics parameters are quantities obtained from nuclides depletion problems, DPT is generally utilized. DPT has been well established in the past $[1,2]$, but there are no computer codes which implement capability of sensitivity calculations based on DPT at present. Theoretical description of DPT is omitted in the present paper, but interested readers can see it in the other papers [1-4]. We have developed our own reactor physics code system CBZ and have implemented DPT-based sensitivity calculation capability into it. On light water reactor analyses, this capability had been limited to single pincell problems originally [3], but it has been extended to multi-cell problems [4]. More recently, DPT has been extended for depletion calculations with the predictor-corrector method, which is mandatory in calculations of actual fuel assemblies including burnable neutron absorbers [5].

\subsection{On linearity of reactor physics parameters to nuclear data}

Although the adjoint-based procedure possess several advantages, it introduces an important assumption: linear dependence of reactor physics parameters with respect to nuclear data. This assumption should be valid if nuclear data uncertainties are small, but it is not the case if these uncertainties are large. Since probability density functions of nuclear data are not explicitly defined in evaluated nuclear data files, the normal distributions are generally assumed. If linearity of reactor physics parameters to 


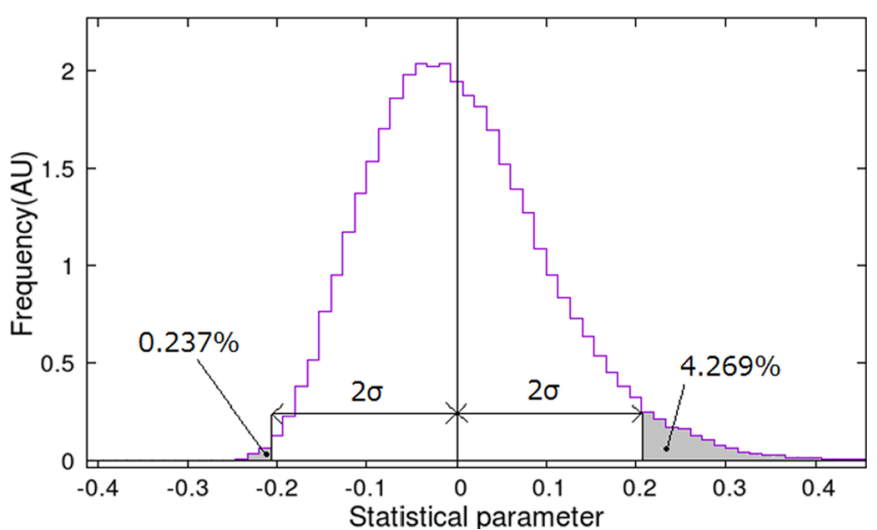

Fig. 1. Example of distorted probability density distribution.

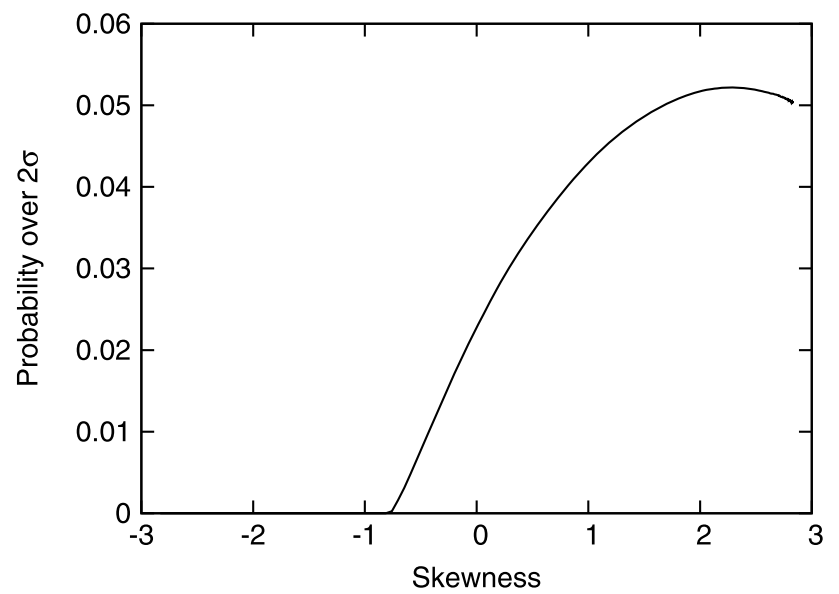

Fig. 2. Relationship between skewness and probability over $2 \sigma$.

nuclear data holds, probability density distribution of reactor physics parameters should be also the normal distribution. On the other hand, if the linearity does not hold, the probability density distribution should be distorted. Figure 1 shows an example of distorted distribution. In this case, probability that a statistical parameter takes value larger than its average plus $2 \sigma$ is around $4.3 \%$, which is around $2.3 \%$ in case of the normal distribution. This might be problematic in parameter specifications with proper margin in nuclear reactor safety analyses. Figure 2 shows relationship between skewness of a probability density distribution and probability that a parameter takes larger value than its average plus $2 \sigma$. This figure is prepared by assuming second-order dependence of an output parameter to an input parameter of the normal distribution.

In our previous study, a linearity check has been done for fission products nuclides generation of light water reactors by using the stochastic-based procedure [6]. This has been accomplished by observing high-order statistical quantities, such as skewness and kurtosis, and deviations from values of the normal distribution are regarded as indices to check the linearity.

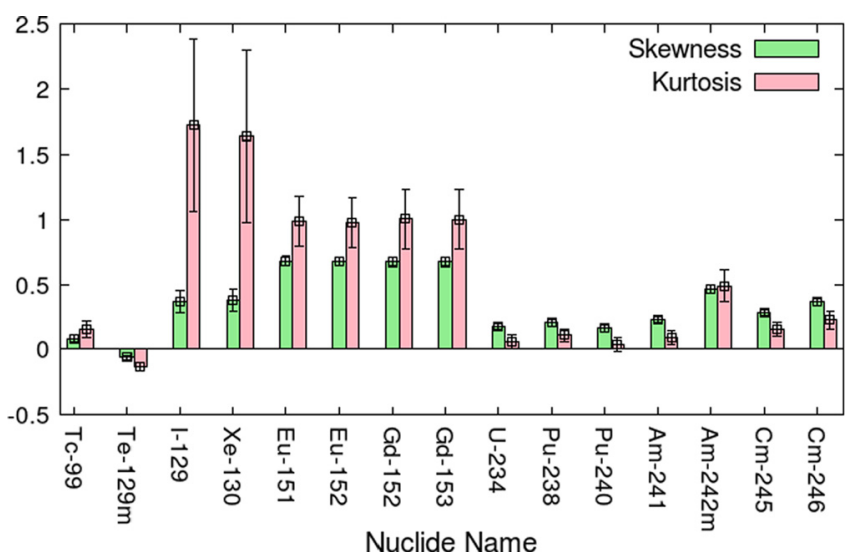

Fig. 3. Skewness and kurtosis of number densities after depletion of several nuclides.

In most nuclides, linearity of number densities after depletion with respect to nuclear data has been confirmed, but some exceptional cases have been observed. Skewness and kurtosis calculated are shown in Figure 3. Error bars are statistical uncertainties estimated by the bootstrap method [7]. It is notable that non-negligible difference in standard deviations between the adjoint-based procedure and the stochastic-based procedure are observed in these number densities because of non-linear effect which can be taken into account only by the stochastic-based procedure. Large skewness of some europium and gadolinium isotopes are caused by large uncertainty of half life of samarium151. Since JENDL/FPD-2011 gives no uncertainty to this nuclear data, we have assumed $100 \%$ uncertainty. This large uncertainty results in non-linear effect to its daughter nuclides generation, and distorted distributions of these nuclides number densities are obtained. It is interesting to point out that if uncertainty is evaluated not to half life but to decay constant, this should not occur because generation of daughter nuclide can be well approximated by the firstorder as

$$
N_{2}(t)=1-N_{1}(0) \exp \left(-\lambda_{1} t\right) \approx N_{1} \lambda_{1} t,
$$

where $N_{i}(t)$ is number density of nuclide $i$ at $t$ and $\lambda_{i}$ is decay constant of nuclide $i$. It is also interesting to point out that this might be totally different if we consider number densities of nuclides which are in an equilibrium state as suggested by Endo [8]. Number density of such a nuclide can be represented as

$$
N(t) \approx \frac{R}{\lambda}=R T_{1 / 2} /(\ln 2),
$$

where $T_{1 / 2}$ is half life. This suggests that number density should be linear on its half life.

\subsection{Uncertainty quantification of decay heat and its reduction by nuclear data adjustment}

Nuclear data-induced uncertainty of decay heat have been quantified with the adjoint-based procedure. In addition, 

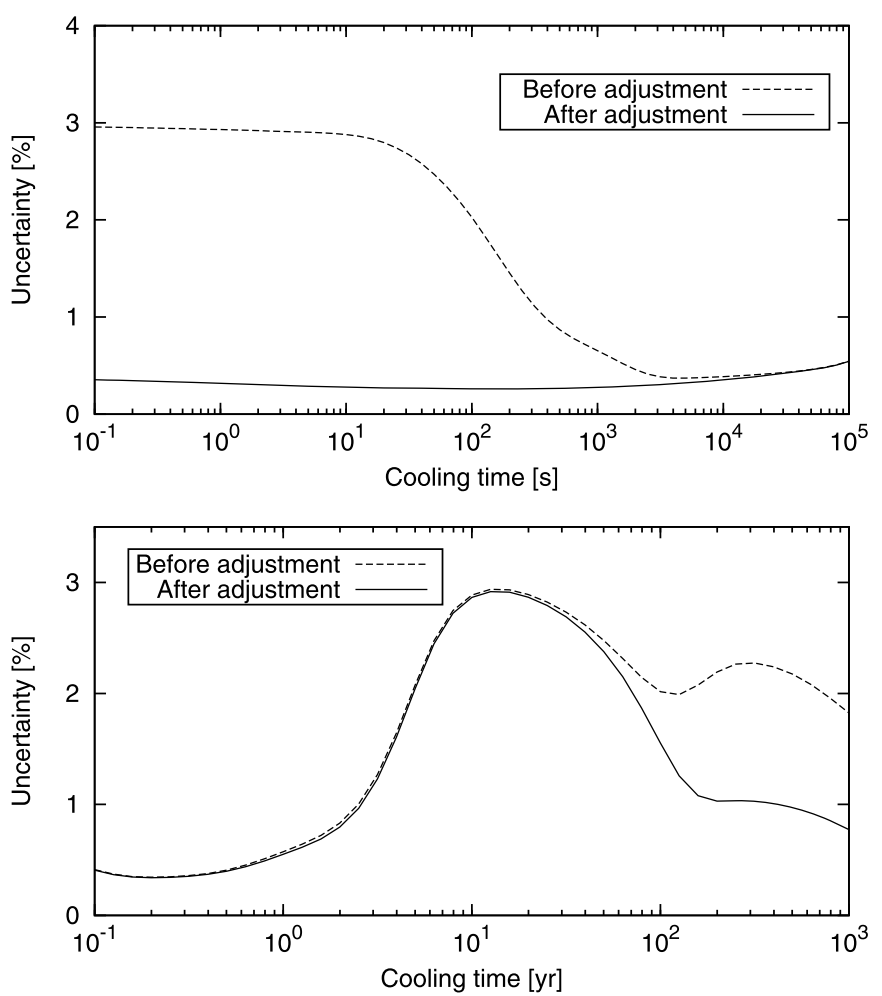

Fig. 4. Nuclear data-induced uncertainty of decay heat. The upper one is the short-term decay heat and the lower is the longterm one.

uncertainty reduction using the nuclear data adjustment method with measurement data has been attempted by our research group [9]. We have quantified uncertainties of both the short-term decay heat, which is important in safety analyses of nuclear reactors, and the long-term decay heat, which is important in management of spent nuclear fuels. Figure 4 shows uncertainties of short-term and longterm decay heat before and after the nuclear data adjustment. We have demonstrated that the uncertainties of the short-term and long-term decay heat can be reduced by using measurement data of decay heat after fission pulse and that of post irradiation examination, respectively.

\subsection{On correlation between decay heat and $\beta$-delayed neutrons emission}

$\beta$-delayed neutrons emission is quite important in nuclear reactors kinetics. We have quantified nuclear data-induced uncertainty of $\beta$-delayed neutron emission rates in the frame of summation calculations [10]. In addition, since nuclear data relevant to the $\beta$-delayed neutrons emission are also important in decay heat calculations, we have examined correlation between nuclear data-induced uncertainty of decay heat and that of $\beta$-delayed neutrons emission [11]. Correlation matrix of decay heat and $\beta$-delayed neutron activity after uranium-235 fission by thermal neutrons is shown in Figure 5. This matrix is decomposed into three blocks: $\gamma$ component of decay heat (from 1 to 31 in $x$ and $y$ axes), (2) $\beta$ component of decay

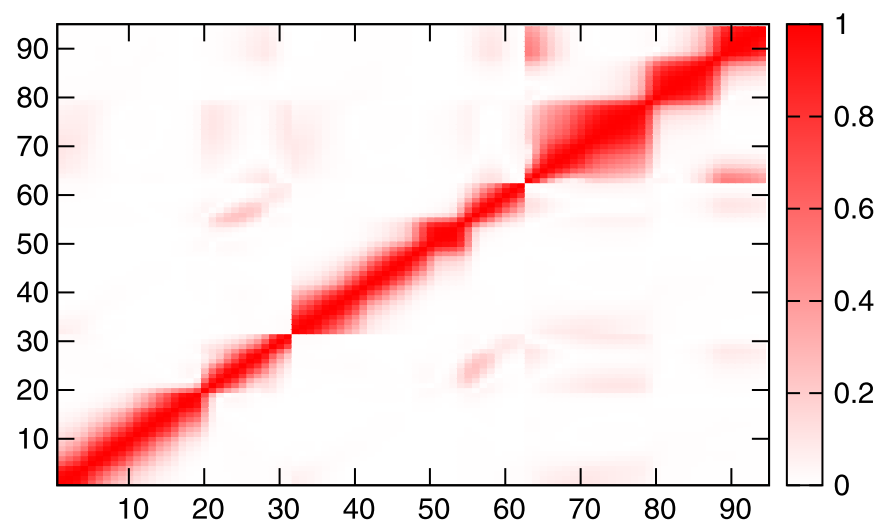

Fig. 5. Correlation matrix of decay heat and $\beta$-delayed neutron activity after uranium-235 fission by thermal neutrons.

heat (from 32 to 62 ) and (3) $\beta$-delayed neutron activities (from 63 to 94). This result suggests that the correlation among these blocks is insignificant, and that independent treatments of decay heat and $\beta$-delayed neutrons emissions are possible.

\section{UQ of decay heat in various conditions with DPT}

By virtue of DPT, we carry out extensive calculations about UQ on decay heat with the code system CBZ. In these calculations, all the fission product nuclides given in evaluated nuclear data files are explicitly treated. Correlation in fission yields data among different nuclides is taken into account by a method proposed by Katakura [12]. Figure 6 shows nuclear data-induced uncertainty of decay heat after uranium-235 fissions with thermal neutrons with different irradiation periods. Decay heat uncertainty after fission burst is almost the same as that after 1-second irradiation, so it is not shown in the present paper. JENDL/FPY-2011 and JENDL/FPD-2011 are used. In these calculations, $100 \%$ uncertainty is assumed to nuclear data to which uncertainty is not evaluated in these files. It can be observed that decay energy uncertainty is dominant in all the irradiation periods. Next, decay heat uncertainty is calculated with an assumption that $0 \%$ uncertainty is given to nuclear data which have no uncertainty information. Results are shown in Figure 7. The uncertainty is significantly reduced from that shown in Figure 6, so it is preferred that uncertainty of decay energy is evaluated somehow in the JENDL library. The same tendency is observed when the ENDF and JEFF libraries are used, but it is less significant than the JENDL case.

Finally decay heat uncertainty is calculated for fuel pincell model of typical light water reactors. Uranium-235 enrichment of this fuel is $4.1 \mathrm{wt} . \%$. Decay heat uncertainty is calculated with different burnup: 1, 5, 10, 20 and $40 \mathrm{GWD} / \mathrm{t}$. Figure 8 shows decay heat uncertainty with several burnup. It is interesting to point out that the decay heat uncertainty is not significantly dependent on burnup, and that it is quite similar with that of finite-period irradiation case shown in Figure 7. 
(a) $1 \mathrm{Sec}$.

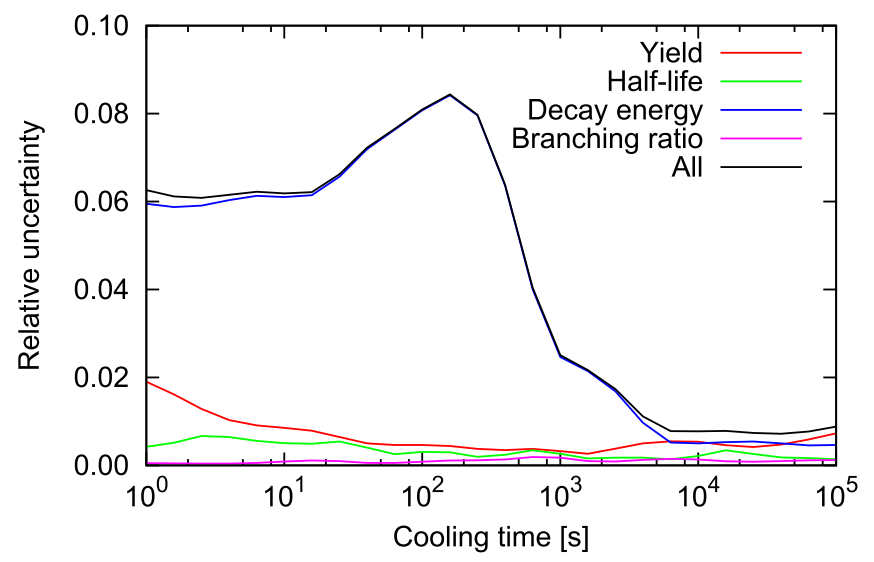

(c) $100 \mathrm{Sec}$.

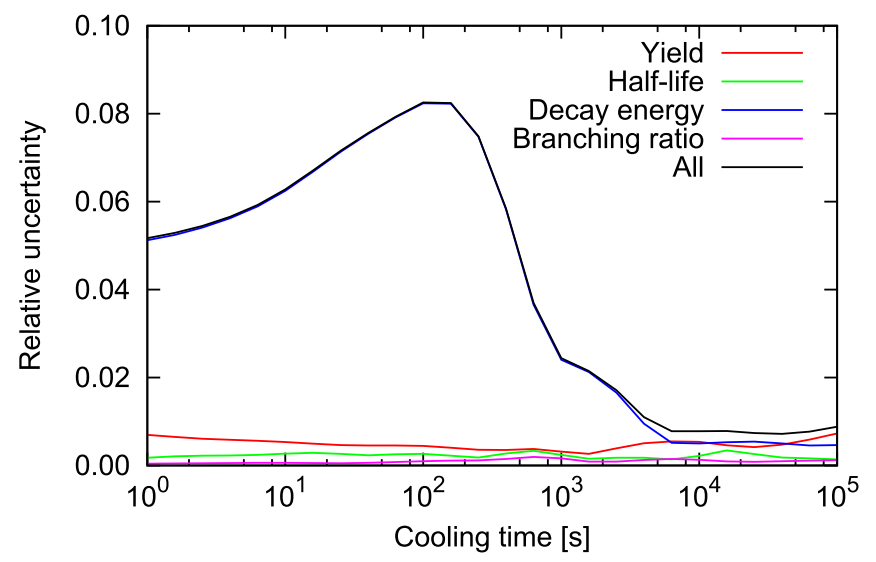

(e) $10000 \mathrm{Sec}$.

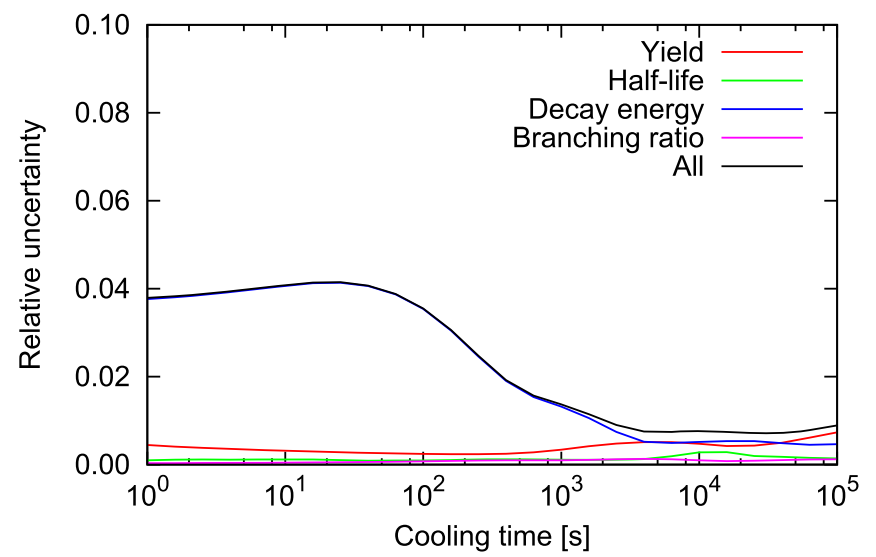

(b) $10 \mathrm{Sec}$.

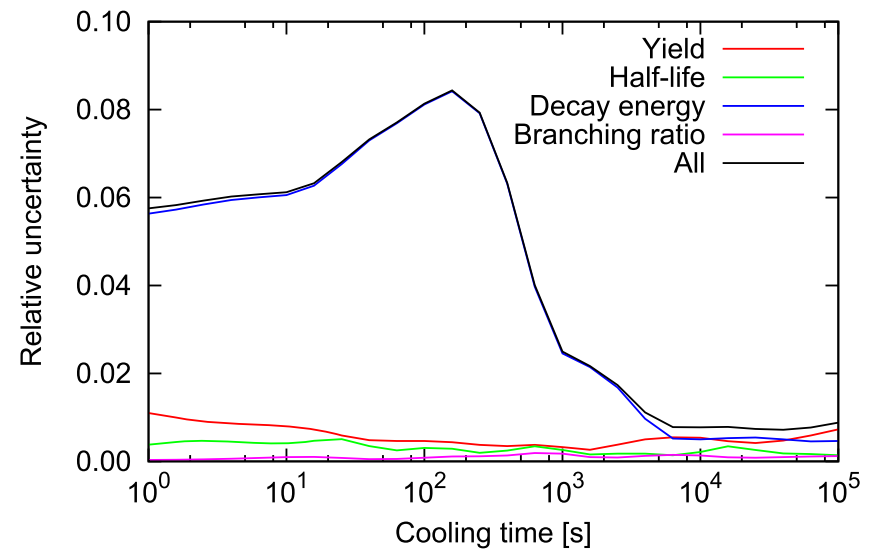

(d) $1000 \mathrm{Sec}$.

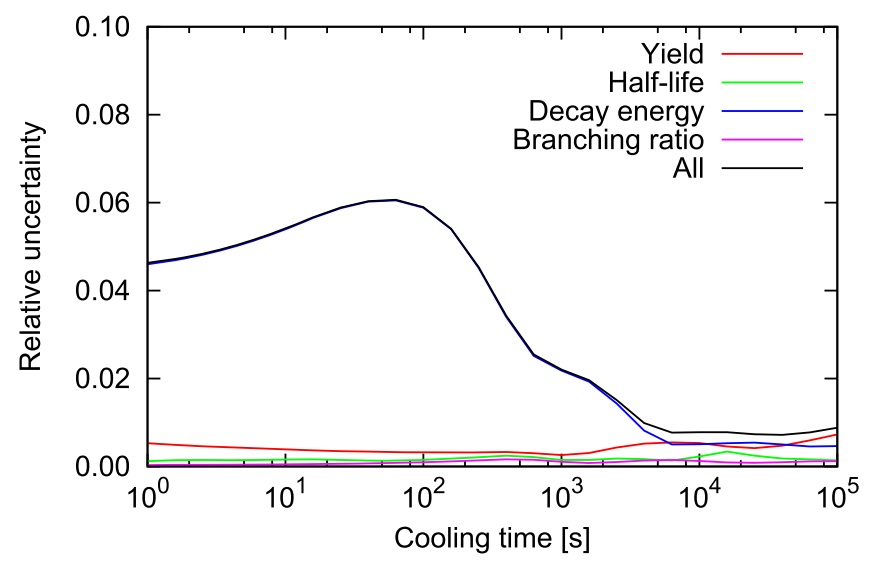

(f) $100000 \mathrm{Sec}$.

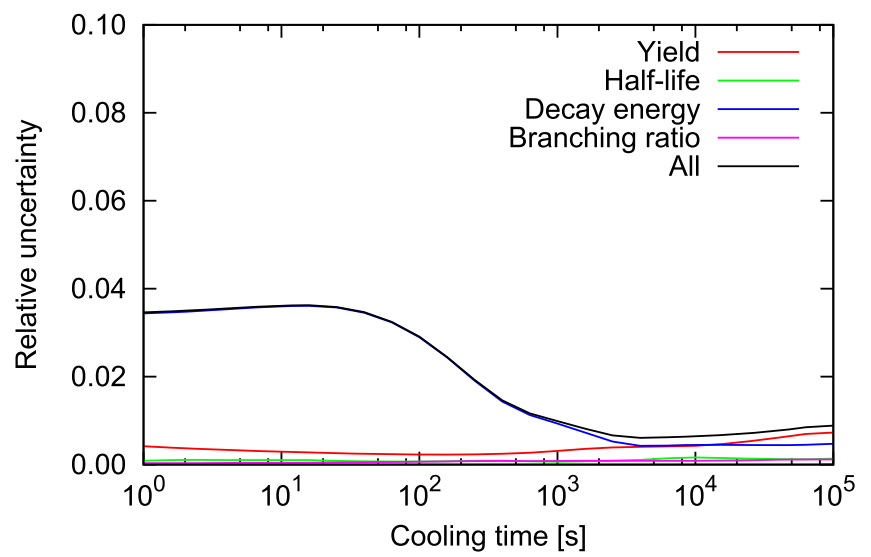

Fig. 6. Nuclear data-induced uncertainty of decay heat after uranium-235 fission with thermal neutrons with different irradiation periods. Default uncertainty is assumed $100 \%$.

Figure 9 shows a correlation matrix of decay heat uncertainties after 100 000-second irradiation and $1 \mathrm{GWD} / \mathrm{t}$ burnup. This matrix is decomposed into two blocks: decay heat after 100000 -second irradiation (from 1 to 26 in $x$ and $y$ axes) and $1 \mathrm{GWD} / \mathrm{t}$ burnup (from 27 to 52 ). In each submatrix, smaller index corresponds to short cooling time. Strong positive correlation can be observed between these two different conditions. 
(a) $1 \mathrm{Sec}$.

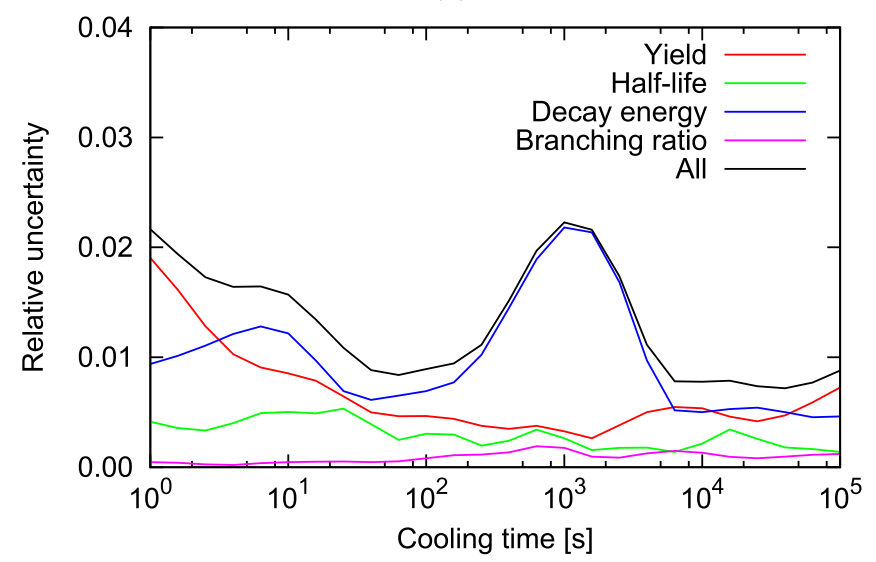

(c) $100 \mathrm{Sec}$.

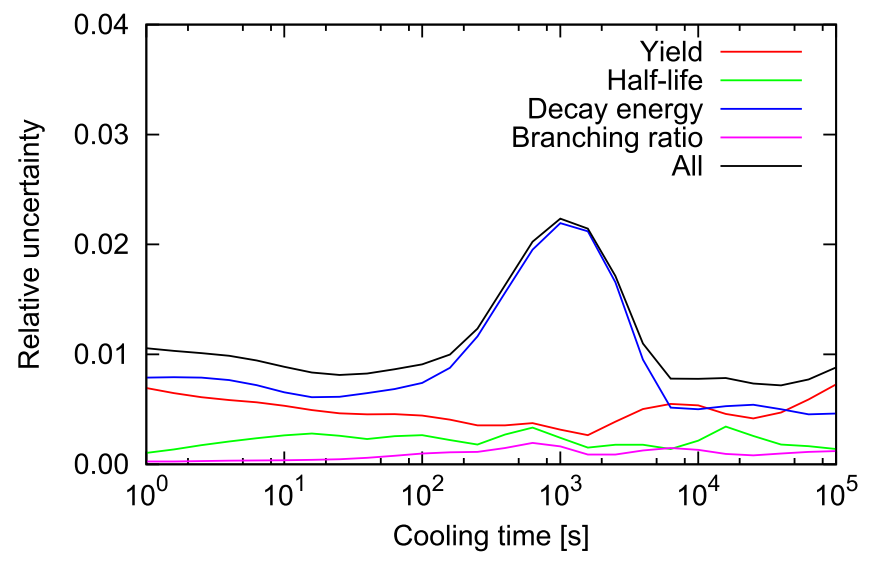

(e) $10000 \mathrm{Sec}$.

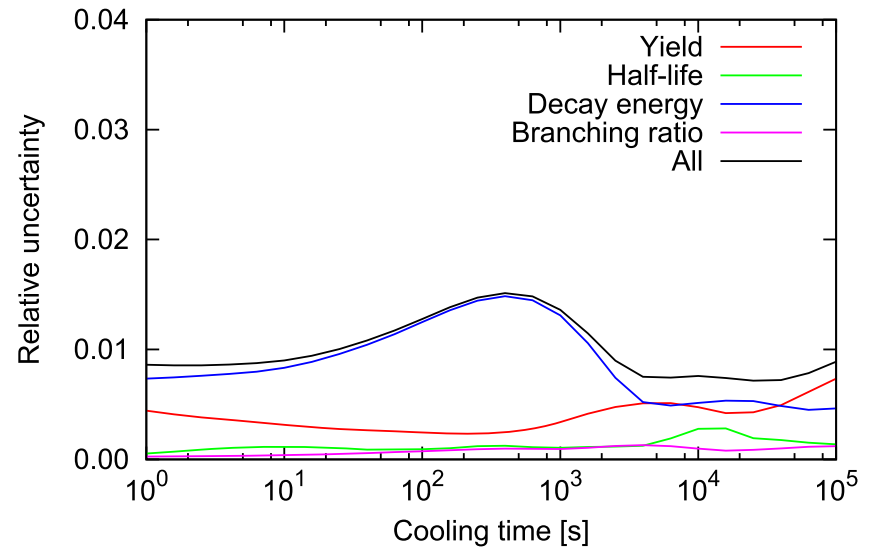

(b) $10 \mathrm{Sec}$

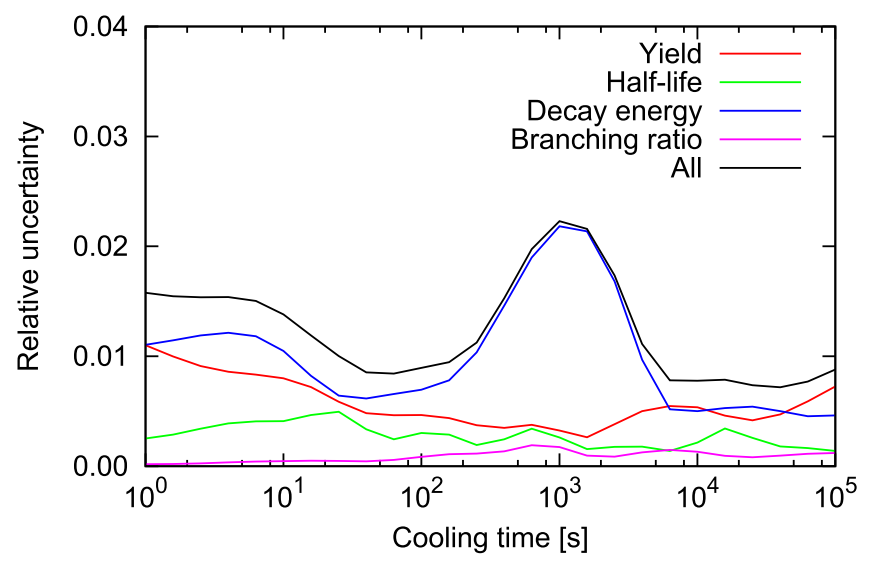

(d) $1000 \mathrm{Sec}$.

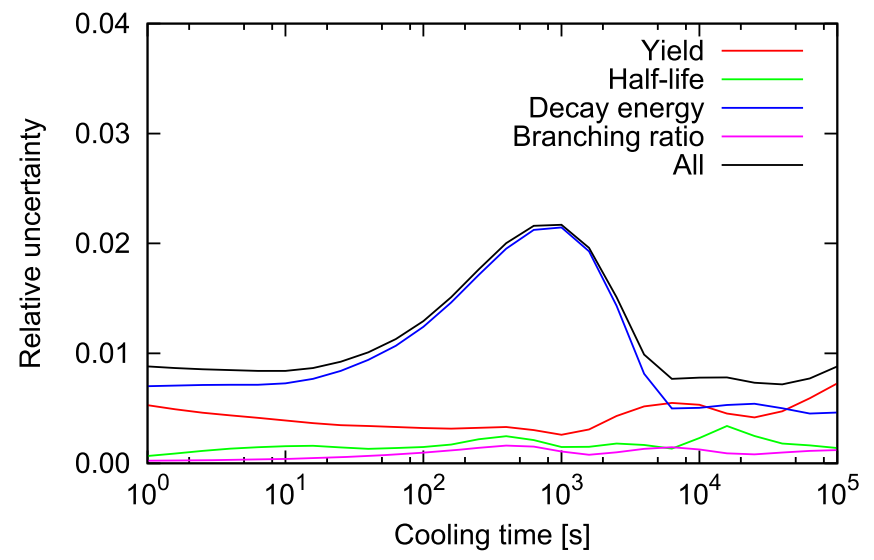

(f) $100000 \mathrm{Sec}$.

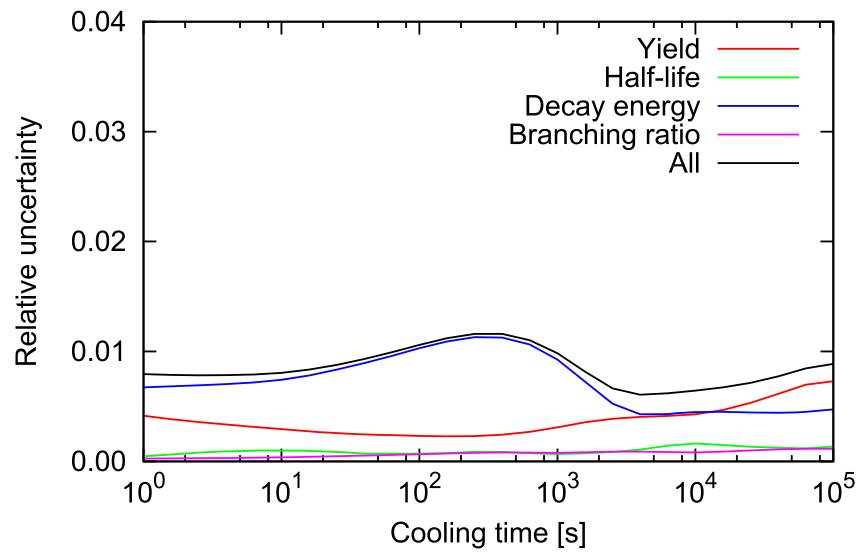

Fig. 7. Nuclear data-induced uncertainty of decay heat after uranium-235 fission with thermal neutrons with different irradiation periods. Default uncertainty is assumed $0 \%$. 
(a) $1 \mathrm{GWD} / \mathrm{t}$

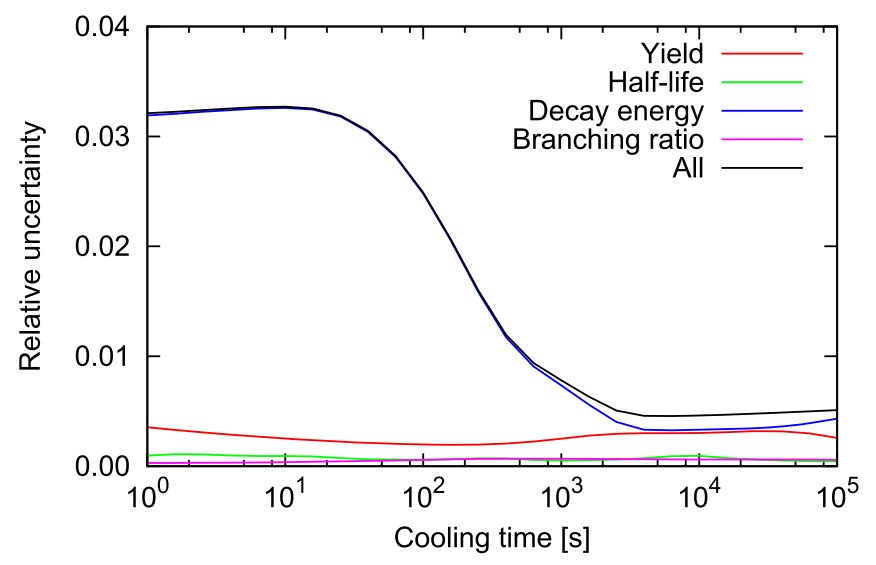

(c) $10 \mathrm{GWD} / \mathrm{t}$

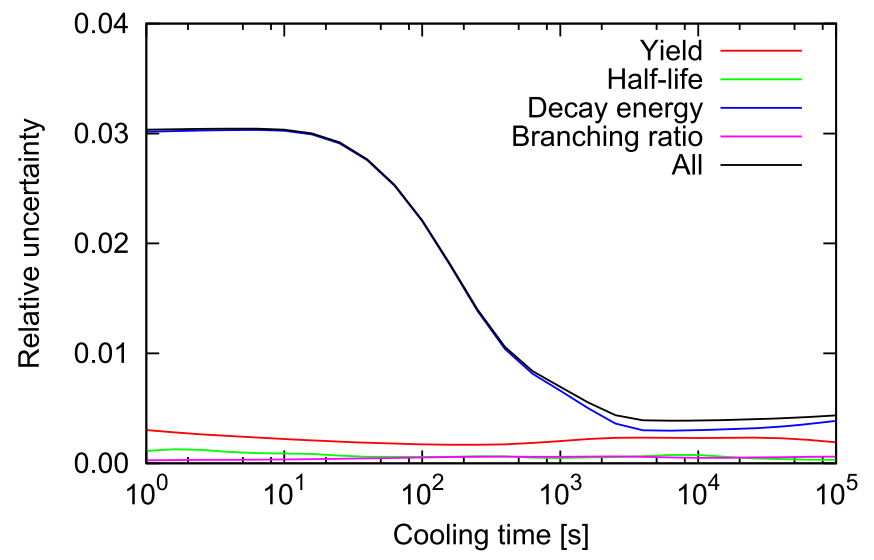

(e) $40 \mathrm{GWD} / \mathrm{t}$

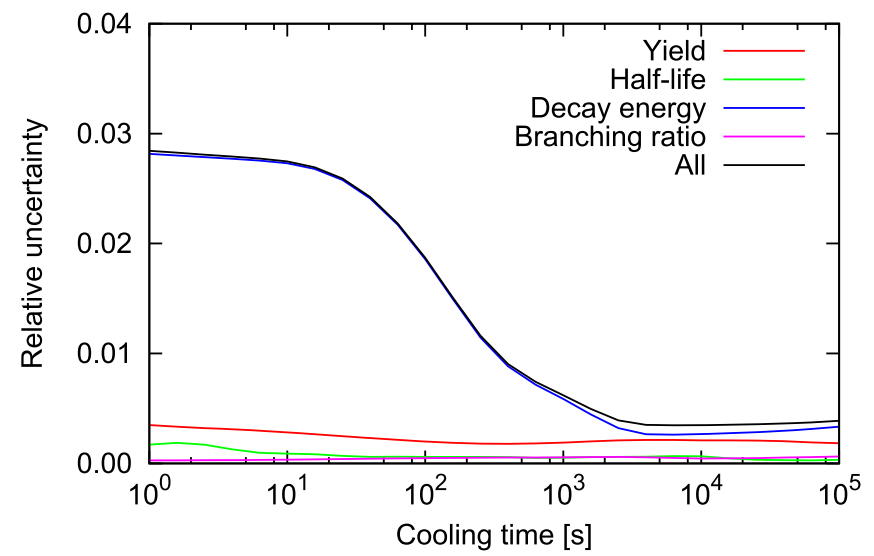

(b) $5 \mathrm{GWD} / \mathrm{t}$

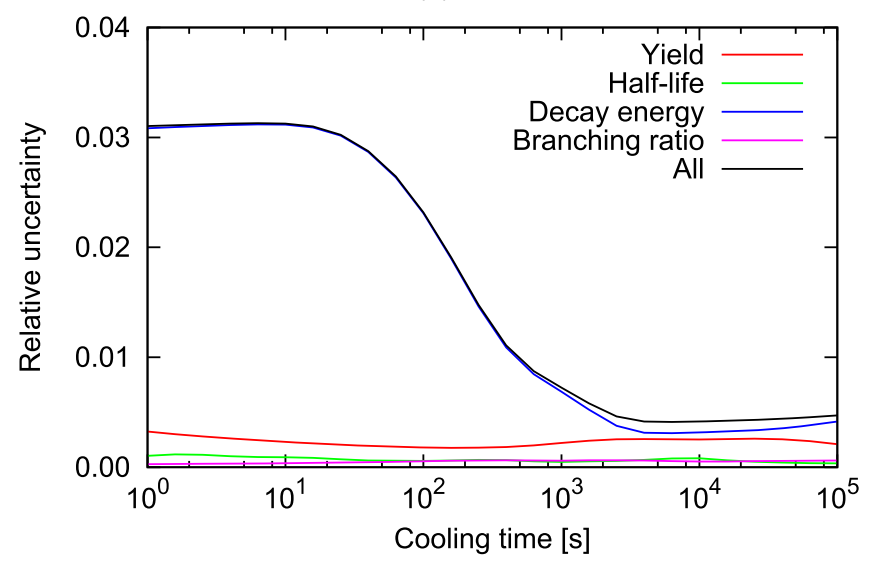

(d) $20 \mathrm{GWD} / \mathrm{t}$

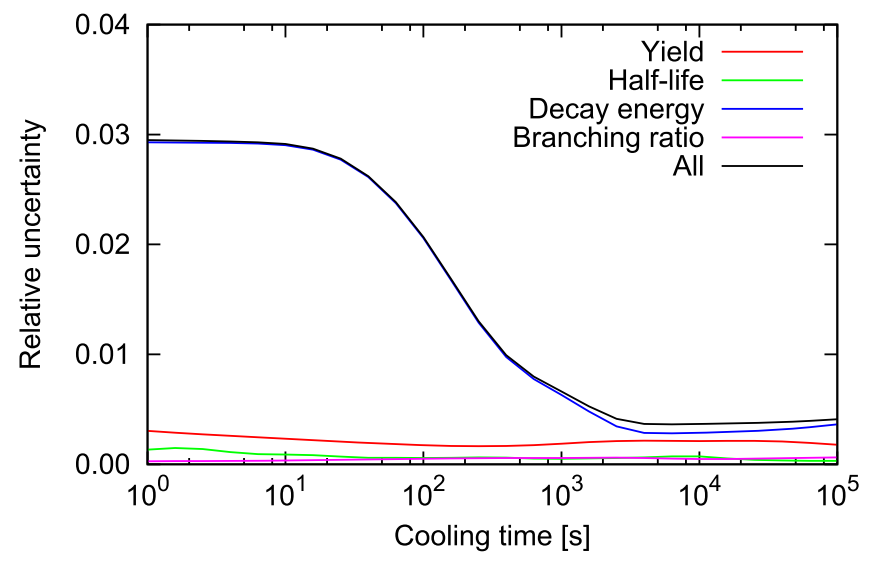

Fig. 8. Nuclear data-induced uncertainty of decay heat after uranium-235 fission with thermal neutrons with different burnup. Default uncertainty is assumed $0 \%$. 


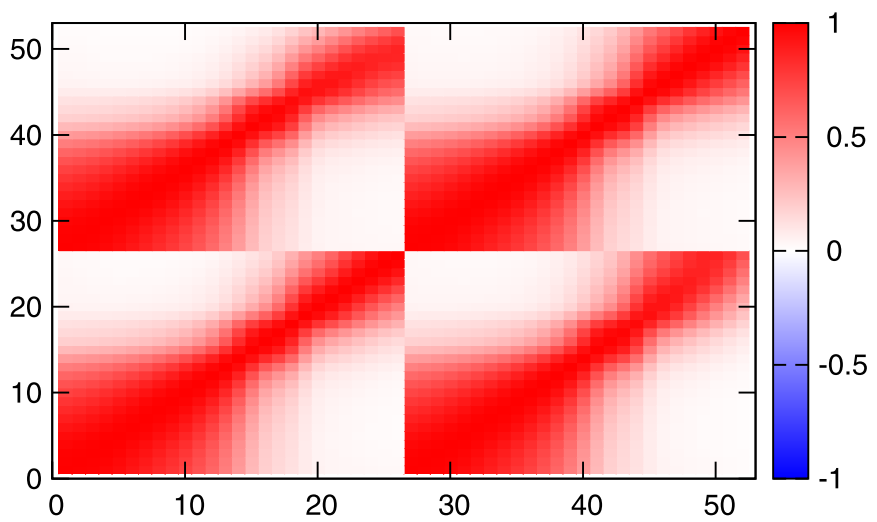

Fig. 9. Correlation matrix of decay heat uncertainties after 100000 -second irradiation and $1 \mathrm{GWD} / \mathrm{t}$ burnup.

\section{Conclusion}

In the present paper, we have reviewed our previous works on UQ of reactor physics parameters. Furthermore, we have carried out extensive calculations about UQ on decay heat with several different numerical conditions by the DPT-based capability of the reactor physics code system CBZ.

\section{References}

1. A. Gandini, Nucl. Sci. Eng. 38, 38 (1969)

2. M.L. Williams, Nucl. Sci. Eng. 70, 20 (1979)

3. G. Chiba, M. Tsuji, T. Narabayashi, J. Nucl. Sci. Technol. 50, $751(2013)$

4. G. Chiba, Y. Kawamoto, T. Narabayashi, Ann. Nucl. Energy 96, $313(2016)$

5. G. Chiba, J. Nucl. Sci. Technol. 55, 450 (2018)

6. S. Nihira, G. Chiba, in Proceeding of Reactor Physics Asia Conference 2017 (RPHA2017), Chengdu, China, August 2425, 2017

7. T. Endo, T. Watanabe, A. Yamamoto, J. Nucl. Sci. Technol. 52, 993 (2015)

8. T. Endo, private communication

9. Y. Kawamoto, G. Chiba, J. Nucl. Sci. Technol. 54, 213 (2017)

10. G. Chiba, Ann. Nucl. Energy 85, 846 (2015)

11. G. Chiba, Ann. Nucl. Energy 101, 23 (2017)

12. J. Katakura, J. Nucl. Sci. Technol. 50, 799 (2016)

Cite this article as: Go Chiba, Shunsuke Nihira, Uncertainty quantification works relevant to fission yields and decay data, EPJ

Nuclear Sci. Technol. 4, 43 (2018) 